$x$ xiii

(7) $y_{1}$ diminishing

(8) $y_{1}$ diminishing by (4) and (6)

(9) $y_{2}$ is - , by (7) by (5) and (6)

(10) $y_{2}$ is - , by (8)

(11) by (9) and (10)

either $y_{2}$ is - or $y_{2}=0$ and is maximum.

Generalising, where $y_{n}$ is a maximum, there $y_{n+1}=0$ and either $y_{n+2}$ is negative or $y_{n+2}=0$ and is maximum. This leads directly to the usual conditions for a maximum

$$
\begin{array}{ll} 
& y_{1}=0, y_{2} \text { is negative } \\
\text { or } & y_{1}=y_{2}=y_{3}=0, y_{4} \text { is negative } \\
\text { or } & y_{1}=y_{2}=y_{3}=y_{4}=y_{5}=0, y_{6} \text { is negative, etc. }
\end{array}
$$

Further the relation between the sign of $d^{2} y / d x^{2}$ and the concavity of an arc is often obscurely presented. Take an $x$ or time axis horizontally and a $y$ axis vertically and consider an arc $A B$ everywhere concave down. Let $C$ be a point on the are such that $A C$ and $C B$ have equal horizontal projections, and let their vertical projections be $a c$ and $c b$. Then algebraically we have from a figure $a c>c b$, that is, heights gained in equal successive times are diminishing and therefore there is a retardation and $d^{2} y / d x^{2}$ is negative. And we similarly associate concavity upwards with positive values of $d^{2} y / d x^{2}$.

Merchiston Crescent,

EDINBURGh.

\title{
On certain modular determinants
}

By H. W. Turnbull.

An interesting determinant occurs in the fifth volume of Muir's History ${ }^{1}$. It is

$$
\Delta=\left.\vdots a_{r s}\right|_{n}
$$

1 Sir Thomas Muir, The History of Determinants, $1900-1920$ (Blackie, 1930), p. 340.

Question 4269. L'Internédiaire des Math., 20 (1913), p. 218, proposed by E. Maillet: reply by E. Malo, 21, pp. 173-176. 
xxiv

where $n=\frac{1}{2}(p-1)$, and $a_{r s}$ is the smallest positive integer such that

$$
r a_{r s}=s(\bmod p),
$$

$p$ being any odd prime number. It is evident that each element $a_{r s}$ is unique and non zero. For $p=5,7,11$ the determinants are

$$
\begin{array}{lll|lll}
1 & 2 \\
3 & 1
\end{array} \quad, \quad \begin{array}{lll}
1 & 2 & 3 \\
4 & 1 & 5 \\
5 & 3 & 1
\end{array}|,| \begin{array}{lllll}
1 & 2 & 3 & 4 & 5 \\
6 & 1 & 7 & 2 & 8 \\
4 & 8 & 1 & 5 & 9 \\
3 & 6 & 9 & 1 & 4 \\
9 & 7 & 5 & 3 & 1
\end{array} \mid
$$

respectively, and their values are

$$
-5,7^{2}, 11^{4} \text {. }
$$

Malo worked out the results up to $p=-13$ inclusive, and suspected that the general result was

$$
\Delta=(-p)^{\frac{1}{(1}(p-3)} \text {. }
$$

Actually this is not the case, although the formula persists until the value $p=29$ is reached. I have checked the results up to $p=31$ and find that the two last cases yield

$$
-8.29^{13} \text { and } 9.3 l^{14}
$$

respectively. The general formula is indeed

$$
q(-p)^{\frac{1}{2}(p-3)}
$$

where $q$ is an integer. Most probably $q$ is a positive integer, but even that surmise is not yet proved.

To prove (4), operate with

$$
\operatorname{row}_{i}-a_{i 1} \text { row }_{1}
$$

on $\Delta$. Let $e_{1}, e_{2}, \ldots, e_{n}$ be the elements of the $i^{\text {th }}$ row, so that the $j^{\text {th }}$ element is now replaced by

$$
\epsilon_{j}-j e_{1}
$$

But, by definition (1), $i e_{j} \equiv j, e_{1} \equiv i(\bmod p)$. Hence $e_{j}-j e_{1} \equiv 0$ $(\bmod p)$, so that $p$ is now a factor of each row below the first. Again by definition $e_{j}$ is either equal to $j e_{1}$ or is less by a multiple of $p$. Hence the new element is either zero or a negative multiple of $p$. This allows the factor $-p$ to be removed from each of $\frac{1}{2}(p-3)$ rows, and expresses $\Delta$ in the form (4), where $q$ is a determinant whose elements are either zeros or positive integers. 
For example, if $p=11$, take the third determinant shewn in (2). The operation (5) yields

$$
(-11)^{4} \mid \begin{array}{ccccc}
1 & 2 & 3 & 4 & 5 \\
\cdot & 1 & 1 & 2 & 2 \\
\cdot & \cdot & 1 & 1 & 1 \\
\cdot & \cdot & . & 1 & 1 \\
\cdot & 1 & 2 & 3 & 4
\end{array}
$$

$=(-11)^{4} q . \quad$ By forming first differences of columns $\left(\mathrm{col}_{1}, \mathrm{col}_{2}-\mathrm{col}_{1}\right.$, $\mathrm{Col}_{3}-\mathrm{col}_{2}$, etc.) this reduces to

$$
\begin{array}{rccccc} 
& 1 & 1 & 1 & 1 & 1 \\
(-11)^{4} & \cdot & 1 & . & 1 & . \\
& . & . & 1 & . & .
\end{array}=11^{4}
$$

so that $q=1$ when $p=11$.

It is easy to prove, from (1), that, on the $i^{\text {th }}$ row of $\Delta$ the elements $e_{1}, e_{2}, \ldots$ rise by a step $e_{1}$ or else fall by a step $\left(p-e_{1}\right)$, while keeping within the non zero values less than $p$. The element $e_{i}$ which is on the leading diagonal is necessarily equal to unity: also the first set of elements, $i$ in number, give the recurrence pattern which is reproduced by the next such set, a unit step higher, and so on. For example, if $p=31$, the fourth row is

$$
8,16,24,1 ; 9,17,25,2 ; 10,18,26,3 ; 11,19,27 .
$$

Operation (5) and then division throughout by -31 yields the row

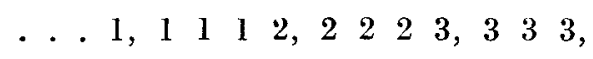

and the first differences by columns produces the recurrence

$$
\text { ... } 1, \ldots 1, \ldots 1, \ldots
$$

It follows that $q$ is now expressed as a determinant $\Delta_{q}$ with $p$ rows and columns, each of whose elements is either 0 or 1 , where the elements on the $i^{\text {th }}$ row recur in cycles of length $i$, for each row. The last element of a cycle is necessarily 1. But it by no means follows that all the others are zeros.

For example the third row has the cycle $0,0,1$ whenever $p$ is a prime of the form $6 r-1$ (cf. $p=11$ above), and the cycle $0,1,1$ when $p=6 r+1$. Again the fourth row has $0,0,0,1$ when $p=4 k+3$, and $0,1,1,1$ when $p=4 k+1$. 
$\mathbf{x x} \nabla \mathbf{i}$

The cycles on the fifth row are

$$
\begin{aligned}
& 00001 \text { for } p=5 r-1) \\
& 01111 \text { for } p=5 r+1 \text { j } \\
& 01011 \text { for } p=5 r-2 \text { ) } \\
& 00101 \text { for } p=5 r+2 j
\end{aligned}
$$

which fall into conjugate pairs; namely, the runs of like symbols (four zeros, then one unit) counted from the left in the case $p=5 r-1$ is the same as the runs (four units, then one zero) in the conjugate cycle, counted from the right, when $p=5 r+1$. $\bmod k$.

In general the cycles on the $k^{\text {th }}$ row are conjugate, for $p= \pm a$

Simple as this determinant $\Delta_{q}$ looks, consisting as it does of zeros and units in recurring cycles on its rows, it is nevertheless quite difficult to compute for high values of $p$. Is $\Delta_{q}$ always positive? or always non zero?

A compact notation is the following

$$
\left\{1,01,0^{2} 1,0^{3} 1,01^{4}\right\}
$$

for the five rowed determinant of formula (7) above, where $0^{r} 1^{s}$ denotes a run of $r$ zeros followed by $s$ units in a cycle of $r+s$ elements. The notation denotes the cycles, and therefore by continuation the rows of $\Delta_{q}$. For example the fourth entry $0^{3} 1$ indicates the cycle 00001 on the fourth row which is therefore $0,0,0,1,0$.

For $p=13$ we find

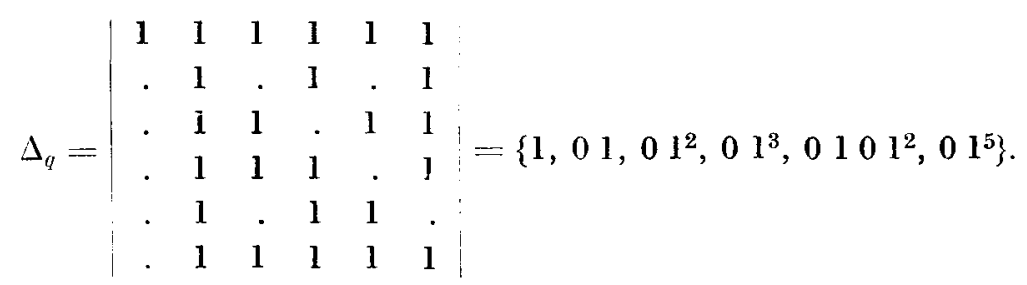

Here $\Delta_{q}=1$. The notation for the fifth cycle indicates a sequence . I, twice, followed by 1 to form a cycle of five elements. The first example in which $\Delta_{q}$ is greater than 1 occurs with $p=29$. The requisite 14-rowed determinant turns out to have the following specification

$$
\begin{gathered}
\left\{1,01,0^{2} 1,01^{3}, 0^{4} 1,0^{5} 1,01^{6},\left(0^{2} 1\right)^{2} 01,0^{2} 1(01)^{3}, 0^{9} 1,\right. \\
\left.\left(0^{3} 1\right)^{2} 0^{2} 1,(01)^{3} 0^{2} 101^{2}, 0\left(0^{2} 1\right)^{4}, 01^{13}\right\}
\end{gathered}
$$


from which $\Delta_{q}$ can be easily constructed. For example, its ninth row, indicated by $\left(0^{2} 1\right)\left(\begin{array}{ll}0 & 1\end{array}\right)^{3}$, is

$$
\text { ..1.1.1.1.1.1, }
$$

where of course the second cycle of nine is incomplete, there being fourteen elements in all. As already stated $\Delta_{q}=8$.

\section{Modular Latin Squares.}

Let each element in (2) be fully reduced to a positive or negative residue nearest to $p$. In fact let $\left|\begin{array}{ll}1 & 2 \\ 3 & 1\end{array}\right|$ be replaced by $\begin{array}{rr}1 & 2 \\ -2 & 1\end{array}$ when $p=5$. In this way we obtain new determinants, whose values are not necessarily equal to the old, but whose structure is more interesting. Apart from sign, each row and each column now contains the first $r$ integers suitably deranged, where $2 r+1=p . \quad$ An array wherein the same $r$ elements occur in each row and column is called a Latin square.

$$
\text { For } p=7 \text { let }\left|\begin{array}{lll}
1 & 2 & 3 \\
4 & 1 & 5 \\
5 & 3 & 1
\end{array}\right| \text { be replaced by } \begin{array}{rrr}
1 & 2 & 3 \\
-3 & 1 & -2 \\
-2 & 3 & 1
\end{array} \text {, and so }
$$

on. On changing the signs of elements throughout the third row and third column we obtain a pure Latin square, $1,2,-3$ occurring in each row and column; but the determinant is zero.

By a suitable rearrangement of rows and columns we can express all these determinants in persymmetric form. For $p=11$ we obtain

$$
L=\mid \begin{array}{rrrrr}
1 & -2 & 4 & 3 & 5 \\
-2 & 4 & 3 & 5 & 1 \\
4 & 3 & 5 & 1 & -2 \\
3 & 5 & 1 & -2 & 4 \\
5 & 1 & -2 & 4 & 3
\end{array}=11^{4}
$$

where, it will be noted, the integers $1,-2,3,4,5$ form a pure Latin square, the negative sign always accompanying the same integer.

Actually this determinant is of simpler structure than the original. It is of a type where the elements of the rows (and columns) form geometrical progressions reduced $\bmod p$ and this gives an order from which the $L$-determinant can at once be written down. Such a progression $1, a, a^{2}, \ldots$ can be formed by taking $a$ to be either $4,-2,5$ or 3 ; and likewise whenever $p=4 r+3$. The resulting $L$-determinants are not essentially distinct for different choices of $a$, 
xxviii

provided that $2 r+1$ is also prime (as when $p=11$ ). Each of them consists of $2 r+1$ rows and columns. Each of the first $2 r+1$ integers occurs in each row, the negative sign always accompanying the same integer. The number of negative signs in a row depends on the value of $p=4 r+3$, but is far from obvious. Actually the row of integers is a complete set of quadratic residues for $p$ : that is, all possible reduced integers $y$ satisfying $x^{2}=y \bmod p$.

When however $2 r+1$ is composite, we may derive Latin squares of smaller size, according to the factors of $2 v+1$. For example, if $p=19,2 r+1=9$. Here -2 is a quadratic residue $\left(6^{2}=-2 \bmod 19\right)$, and the modular geometrical progression, with $a=-2$, is

$$
1,-2,4,-8,-3,6,7,5,9, \bmod 19 .
$$

The sequence recurs after the term 9. With $a=4$ the sequence is also complete, but with $a=-8$ we obtain a progression which repeats. more rapidly,

$$
1,-8,7,1, \ldots \bmod 19 .
$$

The cycle has three terms (three being a factor of $2 r+1$ ). Similar cycles of three terms are $-2,-3,5$ and $4,6,9$, obtained by systematic selection from the complete cycle. Each of these leads to a Latin square, but only the third has a non zero value:

$$
\mid \begin{array}{lll}
4 & 6 & 9 \\
6 & 9 & 4 \\
9 & 4 & 6
\end{array}=-19^{2} .
$$

For the complete Latin square of $2 r+1$ rows and columns, non zero $L$-determinants are comparatively rare. The first few cases are when $4 r+3=p=11,19,43$, for the other cases $p=3,7,23,31$, the sum of the different residues is zero, so that the determinant vanishes by adding all its rows to the first. As before it is easy to shew that the general value of such a determinant is that of (4) above, but in. this case $q$ can be zero and possibly negative.

The evaluation when $p=19$ suggests a procedure:

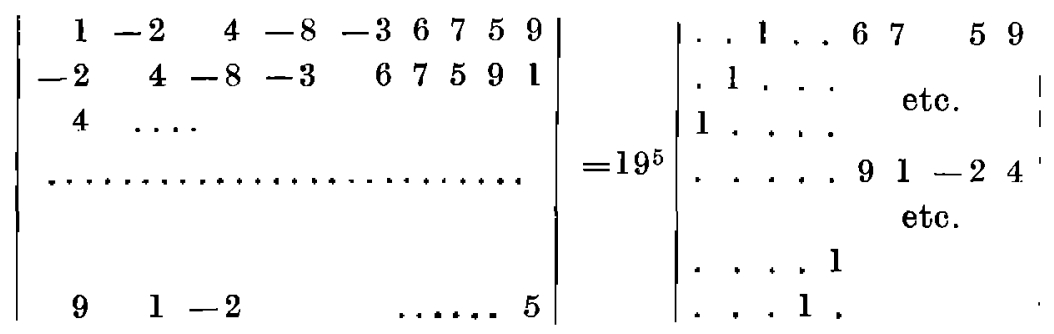


by $\operatorname{col}_{1}-\operatorname{col}_{2}+\operatorname{col}_{5}, \operatorname{col}_{2}-\operatorname{col}_{3}+\operatorname{col}_{6}$, etc. This gives

$$
19^{5}\left|\begin{array}{rrrc}
9 & 1 & -2 & 4 \\
1 & -2 & 4 & -8 \\
-2 & 4 & -8 & -3 \\
4 & -8 & -3 & 6
\end{array}\right|=19^{5}\left|\begin{array}{rrrr}
9 & 1 & -2 & 4 \\
1 & -2 & 4 & -8 \\
. & . & . & -19 \\
. & . & -19 & .
\end{array}\right|=19^{8}
$$

The next non zero case is when $p=43$, which gives a 21-rowed determinant of which the top row is

$$
\begin{gathered}
1,-3,9,16,-5,15,-2,6,-18,11,10,13,4,-12,-7,21, \\
-20,17,-8,-19,14,
\end{gathered}
$$

and the second row starts with -3 , and so on. By bringing all other columns three places to the left and placing the first three columns last, we obtain a form which is easier to handle: $16,-5, \ldots$, $14,1,-3,9$.

By $\operatorname{col}_{1}+\mathrm{col}_{2}-\mathrm{col}_{7}, \mathrm{col}_{2}+\mathrm{col}_{3}-\mathrm{col}_{8}$, etc., the factor 43 appears in each of the first fifteen columns. Also $\mathrm{col}_{j}+\mathbf{3} \mathrm{col}_{j-1}$ applies to the last five columns and produces the factor $43^{5}$. The final result I find to be

$$
\Delta=-4.11 \cdot 727.43^{20} \text {. }
$$

A non zero three-rowed Latin square exists for $p=43$ :

$$
\left|\begin{array}{lll}
10 & 16 & 17 \\
16 & 17 & 10 \\
17 & 10 & 16
\end{array}\right|=-43^{2}
$$

The case when $p=4 r+1$ leads to a kind of skew Latin square. For instance when $p=13$, we have the modular geometrical progression

$$
[1,2,4,8,16,32]=[1,2,4,-5,3,6] \bmod 13 .
$$

This leads to a further type of determinant

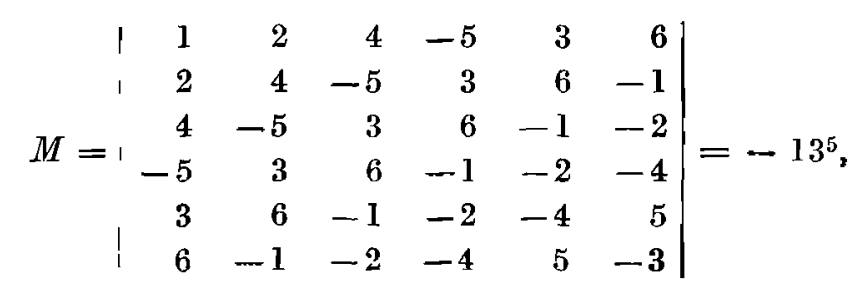

where it will be seen that the signs of the digits are changed when they occur on a new parallel diagonal. 
In this case one two-rowed skew Latin square minor always exists, owing to the fact that $p=4 r+1$ can be expressed as the sum of two squares. Here

$$
\left|\begin{array}{rr}
2 & 3 \\
3 & -2
\end{array}\right|=-\left(2^{2}+3^{2}\right)=-13
$$

The quadratic residues for $p=4 r+1$ always occur in pairs $\pm y$ and involve only half of the possible set of reduced integers: they may or may not lead to a non zero minor determinant. For $p=13$, the six residues are $\pm 1, \pm 3$, \pm 4 : for $p=17$ the eight residues are $\pm 1, \pm 2, \pm 4, \pm 8$. We have

$$
\begin{array}{rrr}
1 & 4 & 3 \\
4 & 3 & -1 \\
3 & -1 & -4
\end{array}|=0,| \begin{array}{rrrr}
1 & 2 & 4 & 8 \\
2 & 4 & 8 & -1 \\
4 & 8 & -1 & -2 \\
8 & -1 & -2 & -4
\end{array} \mid=17^{3} .
$$

The University,

St Andrews.

\section{Inequalities for Positive Series}

By C. E. WALSh.

$$
\text { Let } \begin{aligned}
f(x) & \equiv(1-x)^{b}+b \alpha^{b-1} x \\
\phi(x) & \equiv x^{c}-c \beta^{c-1} x
\end{aligned}
$$

where $b \geqq 1, c \geqq 1,0 \leqq \alpha \leqq 1,0 \leqq \beta \leqq 1$, and $x$ is assumed to lie in the range $(0,1)$. By differentiation, or otherwise, it is easily shewn that $f(x)$ and $\phi(x)$ have minima when $x=1-\alpha$ and when $x=\beta$, respectively. Hence

$$
\begin{aligned}
& (1-x)^{b}+b a^{b-1} x \geqq b a^{b-1}+(1-b) \alpha^{b} \\
& x^{c}-c \beta^{c-1} x \geqq(1-c) \beta^{c} .
\end{aligned}
$$

Multiplying the first of these by $c \beta^{c-1}$, the second by $b a^{b-1}$, and adding, we get

$$
\begin{aligned}
c \beta^{c-1}(1-x)^{b} & +b a^{b-1} x^{c} \geqq b \alpha^{b-1} \beta^{c-1} \\
& +b(c-1) a^{b-1} \beta^{c-1}(1-\beta)+c(1-b) a^{b} \beta^{c-1} .
\end{aligned}
$$

Dividing across by $\beta^{c-1}$, this is the same as

$$
c(1-x) \geqq b x^{b-1}\left(1-\beta^{1-c} x^{c}\right)+b(c-1) \alpha^{b-1}(1-\beta)+c(1-b) \alpha^{b}
$$

